\title{
Late Failure of a Polyethylene Unicompartmental Knee Insert - Retrieval Analysis Protocol and Results
}

\author{
RODICA MARINESCU ${ }^{1 *}$, DAN LAPTOIU ${ }^{2}$, IZABELA-CRISTINA STANCU ${ }^{3}$, \\ CRISTINA BUSUIOC ${ }^{4}$ \\ ${ }^{1}$ University of Medicine and Pharmacy "Carol Davila" Bucharest, Department of Orthopedics, 37 Dionisie Lupu Str., 020021, \\ Bucharest, Romania \\ ${ }^{2}$ Colentina Clinical Hospital, 19 - 21 Stefan cel Mare Str., 020125, Bucharest, Romania \\ ${ }^{3}$ Advanced Polymer Materials Group, University Politehnica of Bucharest, Faculty of Applied Chemistry and Materials \\ Science, 1-7 Gh. Polizu Str., 011061, Bucharest, Romania \\ ${ }^{4}$ University Politehnica of Bucharest, Science and Engineering of Oxide Materials and Nanomaterials Department, 1-7 Gh. \\ Polizu Str., 011061, Bucharest, Romania
}

\begin{abstract}
This study reports the investigation of a degraded polyethylene insert retrieved after the catastrophic failure of a mobile bearing knee implant, occurred after a traumatic event and leading to a late revision. Understanding wear mechanisms of polyethylene components is important to improve the implants for joint replacement. This model of unicompartmental arthroplasty, due to its mobile, congruent insert and design, has the potential to attain low rates of wear, leading to an improved survival of the prosthesis over fixed-bearing knee implants. During the surgical technique, however, it is critical to avoid any source of impingement or incongruent articulation, as this are associated with increased rates of polyethylene wear and may contribute to the early necessity for revision surgery. The investigated insert presented a macroscopic fragmentation, surface and bulk defects and debris. Characterization of the retrieved polyethylene implant fragments was performed by macroscopic examination, by scanning electron microscopy and Fourier transform infrared spectroscopy. Due to a systematic retrieval analysis according to current standards, we can question that, in our case, third body wear is rarely pure bone or pure cement debris related but a combination of the two mentioned. The combination enhanced contribution of polyethylene debris in severe wear initiation and progression. Complete examination of the explants exhibiting breakage or severe wear may help in understanding pathogenic ways of failure in unicompartmental knee.
\end{abstract}

Keywords: mobile bearing insert, unicompartmental knee, failure analysis, retrieval analysis, wear

\section{Introduction}

Joint arthroplasty in cases of severe osteoarthritis is typically performed using implants that replace and restore the joint structure. Knee reconstruction generally involves the total or compartmental replacement of the damaged anatomic structures with a system composed from metallic femoral and tibial components and a polyethylene (PE) insert. Ultra-high molecular-weight polyethylene (UHMWPE) is a synthetic polymer used as standard for the fabrication of inserts in total knee arthroplasty (TKA) or unicompartmental knee arthroplasty (UKA). The use of PE is mainly justified by a structural bio-inspired approach to reconstruct the joint providing an articular surface microstructurally similar to the natural one; the similarity is achieved with a combination of amorphous and semicrystalline phases, resembling the hyaline cartilage [1]. While TKA represents the replacement of the entire knee joint, UKA refers to the replacement of a part of it, generically named compartment.

UKA is a valuable choice in the treatment of the knee medial osteoarthritis [2,3]. In use from 1982, the indications for the mobile bearing unicompartmental have expanded lately as well as the reported survival rates $[4,5]$. Decreased perioperative morbidity, restoration of a more natural gait pattern and a better range of motion (ROM) are among documented advantages of unicompartmental knee over total knee arthroplasty (TKA) [6]. When making the choice for such an implant, the surgeon has to decide

*email: rodicamarinescu@ymail.com 
between mobile and fixed bearing, taking into account case particularities as well as his own personal preferences and training [7]. Among mobile unicompartmental knees, Oxford (Zimmer Biomet ${ }^{\mathrm{TM}}$ ) implant is one of the most used. Not only it is a product in which 3 generations of design and implants were developed, with the latest generation of argon packaged compression molded polyethylene ArCom ${ }^{\circledR}$ (Zimmer Biomet), but also continuous improvements were made for increasing the accuracy of the surgical technique. The PE bearing is highly congruent and it is presumed that, consequently, the wear is reduced and implant survival rates improved. However, several complications are reported related to its clinical usage - progression of osteoarthritis in the contra-lateral compartment, aseptic loosening, infection, bearing dislocation and wear [6]; one of the least frequent complications is bearing fracture, with a reported incidence about $1 \%$ of the cases [7]. Retrieval analysis of such implants is important since it improves understanding on implant behavior and failure mechanism. UKA is a procedure with continuous evolution of products and procedures; different designs were developed and the PE inserts may be either fixed or mobile. In conventional implants, the PE insert is fixed on the tibial tray and its femoral surface is in direct contact with the moving femoral surface while the tibial side is mechanically stable on the tibial component. The mobile PE inserts are freely moving between the femoral and tibial metallic components and therefore, unlike the conventional ones, their tibial face is also moving on a metallic surface. The degradation of polyethylene (PE) components represents an important phenomenon whose understanding and control strongly impact on the patient well-being and implant survival. Oxidation during or post sterilization, delamination, debris formation with local osteolysis are among the main events associated to PE degradation $[1,8]$.

In this study, macroscopic, morphologic and structural characterizations were performed to describe the degradation of the PE insert of a UKA implant after 12 years of use.

\section{Material and methods}

We present the case of a 63-year-old male who underwent UKA with Phase III Oxford [Biomet] in 2007 for antero-medial osteoarthritis. At a late follow-up visit,12 years after surgery, he presented with complaints of pain and disability, in evolution for almost one year, after a traumatic event (accidentally missed a step). At clinical examination a medium effusion was detected on the right knee, ROM was between 20 to 90 degrees of flexion; a strange noise accompanied every knee movement. Radiological examination documented a posterior dislocation of meniscal bearing, while femoral and tibial component looked well fixed (Figure 1a).

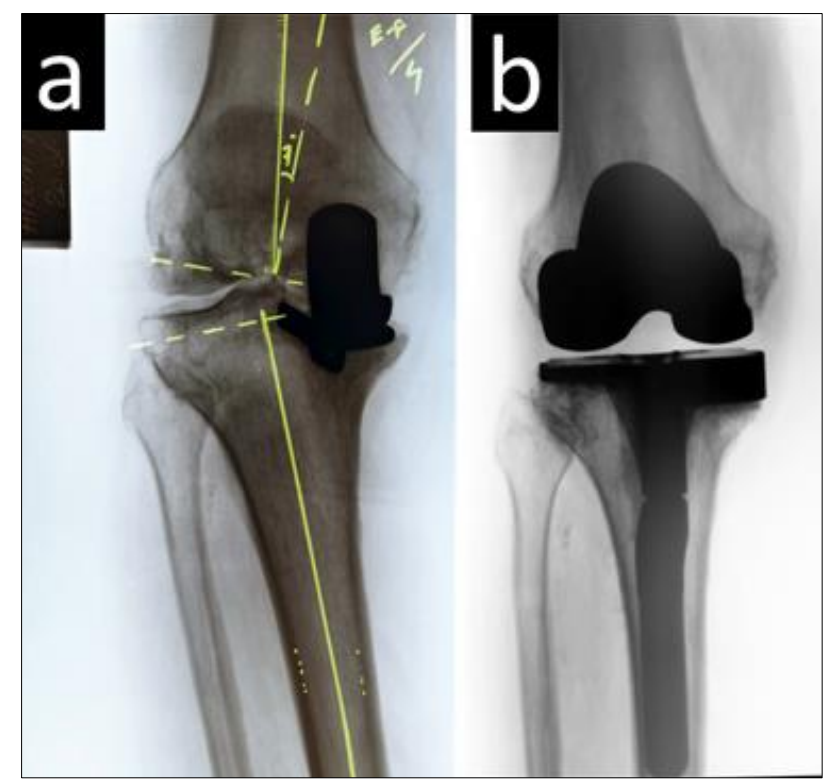

Figure 1. Pre (a) and postoperative (b) X rays at revision time 
The laboratory examination evidenced no infection signs. The indication was revision surgery; synovial samples were harvested from the knee joint and sent for culture, which came out sterile. The meniscal bearing was found broken into two large pieces with an additionally small one. At examination, the femur and tibial plateau exhibited scratches. Together with the severe wear seen on the meniscal bearing this sustained the surgical indication for revision - conversion to a total knee. The conversion was done with a primary postero-stabilised total knee with a different bearing (compression-molded polyethylene with no added calcium stearate, gamma irradiated in an inert nitrogen environment, helping minimize oxidation and creating a more durable, abrasion resistant material - Zimmer Biomet NexGen), completed with a half wedge titanium augment and a short tibial stem (Figure 1b). The rehabilitation was uneventful and the patient returned to full range-of-motion at 6 weeks. According to our standard practice protocol, following ASTM F561 guidance [10], the retrieved meniscal bearing was sent for examination at Politehnica University of Bucharest, with both the femoral and tibial implant:

\subsection{Sample preparation for analysis}

After the surgical revision procedure, the blood and all tissue debris on implants were cleaned using an anionic detergent cleaning solution; after that implants were placed in a disinfection solution in order to be completely free of any biological risk. After drying, the implants were separately wrapped in surgical towels, in order to prevent scratching or other surface modification.

\subsection{Morphological and microstructural analysis}

Morphological and microstructural analysis of polyethylene components was performed by scanning electron microscopy (SEM). A Quanta Inspect F microscope (FEI Company, Hillsboro, OR, USA) equipped with an energy-dispersive X-ray spectroscopy (EDX) probe was used, at $30 \mathrm{kV}$ accelerating voltage, $10 \mathrm{~mm}$ working distance). Samples were gold-sputtered for $40 \mathrm{~s}$ prior to analysis.

\subsection{Fourier-transform infrared spectroscopy (FTIR) analysis}

The PE insert as well as small fragments identified in its fractured areas were characterized using a Jasco 4200 FTIR spectrometer equipped with a Specac Golden Gate attenuated total reflectance (ATR) device, in the 4000-600 $\mathrm{cm}^{-1}$ wave-number region, overlapping 100 scans and with a resolution of $4 \mathrm{~cm}^{-1}$. The fragments (including dark colored debris) were carefully removed from the fractured area using tweezers and analyzed without further washing. Small samples were removed from representative areas of each insert using a clean razor and tweezers and were tested as such, exploring both the surface and the bulk region. ATR-FTIR spectra were recorded also for cement fragments from the retrieved femoral component, as control. To evaluate protein adsorption, surface contamination with bone fragments or potential oxidation of PE insert, FTIR spectra were recorded on distinct regions (at the surface and into the bulk area).

\section{Results and discussions}

Understanding wear mechanisms of PE components is important to improve the implants and techniques for joint replacement [11]. The investigation of wear is typically performed in vivo by radiographic measurements [12], or gravimetrically after implant retrieval - through volumetric and optical inspection $[13,14]$. While such methods are mainly used when pre-wear implants are available for comparison, other methods are needed when such control data is not available for the retrieved prostheses [15]. In the present work we investigated one failed PE component from unicompartmental (partial) knee replacements retrieved during revision surgery.

\subsection{Morphological and microstructural investigation}

The worn and delaminated areas of PE inserts were macroscopically visible (Figure 2). The femoral and tibial metal components are showed to guide the observer in understanding the interaction of the PE components with the metallic ones. While PE mass loss obviously occurred, as macroscopically visible, 
we did not have the possibility to quantify it. However, at optical inspection, the mass loss may be appreciated to be stronger from the femoral side when compared to the tibial side.

In addition, the insert edges and the edges of the fractured areas presented a dark color and small fragments suggesting a potential contamination with migrated materials (indicated with white rectangles in Figure 2 - PE insert). The PE-contacting faces of the metallic tibial and femoral components present worn areas (Figure 2). Furthermore, it can be noticed that wear areas were detected on both sides of the plastic insert, due to its movement on the metal components, with main fractures along the movement direction. The main types of PE degradation are described as 7 modes of damage: scratching, pitting, burnishing, surface deformation, abrasion, exfoliation and bone-cement debris [9]. The investigated sample presented PE fragmentation visible as flake-like fragments, multiple scratch marks and presence of brown fragments in the inner layers of fractured areas (Figure 2 - PE insert). Stronger subsurface whitening was visible on the femoral side when compared to the tibial one. The femoral side view in Figure 2 allowed to observe an almost continuous" white band" close to the edge of the broken PE pieces. Such appearance is considered as an indicator of oxidation of PE, aspect typically associated with long implantation times [16].

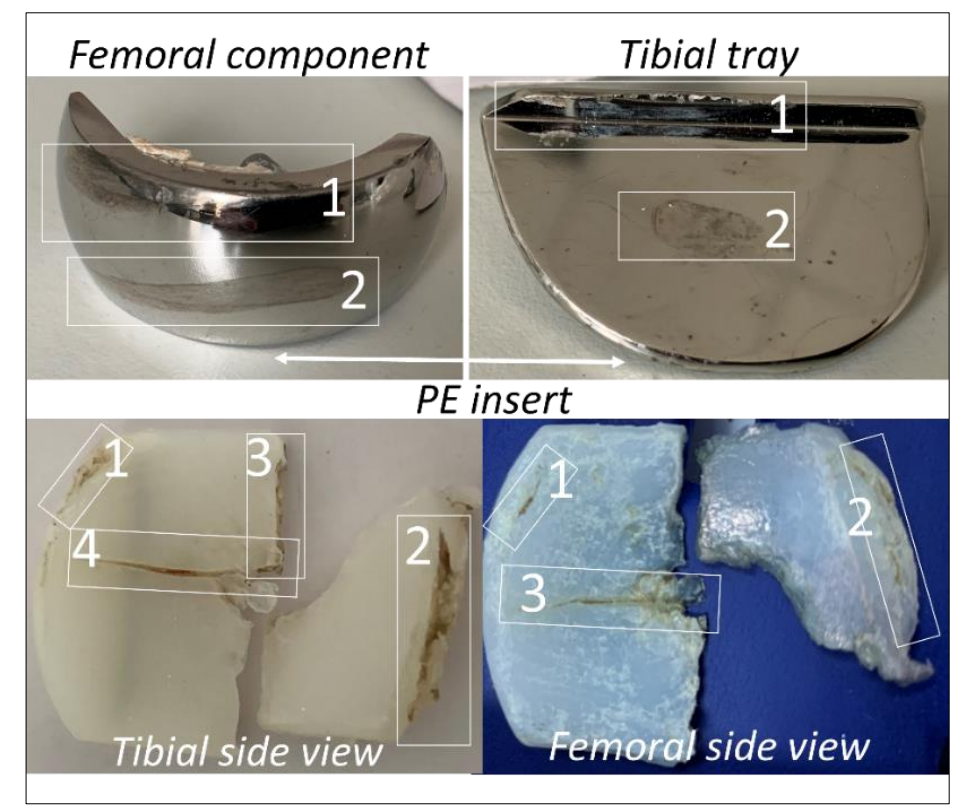

Figure 2. Qualitative assessment. Digital photographs of the components of the retrieved unicompartmental knee implants, from left to right: femoral component, with 2 visible degraded areas (white rectangles 1 and 2); tibial tray with a median metal

degradation (red rectangle 1) and a central metal degradation (white rectangle 2).

The direction of the movement of the femoral head is indicated in white arrow.

Retrieved polyethylene (PE) insert consisting in two large fragments: tibial side view - the PE liner lost its integrity, 4 main areas with intense degradation and contamination

with particles; articular face contacting the femoral component (femoral side view) the PE liner lost its integrity and present severe degradation, white rectangles indicate the

3 main areas with intense degradation and contamination with particles

SEM analysis indicated common wear patterns such as fractures, pitting, and scratches. Figure 3a is representative for a high surface damage consisting in fracture, pitting, scratches and mass loss on the femoral side of the insert. The morphology of some micro particles (around $10-30 \mu \mathrm{m}$ ) distinguishes from the degraded polymer matrix, as visible in secondary electrons in micrographs c-f from Figure 3. When examined in back scattered electrons (BSED), these fragments appear to present a distinct composition; they are denser when compared to the PE matrix, as visible in the micrographs c' - $\mathrm{f}^{\prime}$ (Figure 
3). Such morpho-structural appearance suggests foreign fragments that might be assigned to migrated bone or bone cement. Figure $3 \mathrm{~b}$ presents a representative EDAX spectrum recorded for one of these particles. The detection of calcium and phosphorus peaks suggests a phospho-calcic phase that may be representative for a bone fragment.

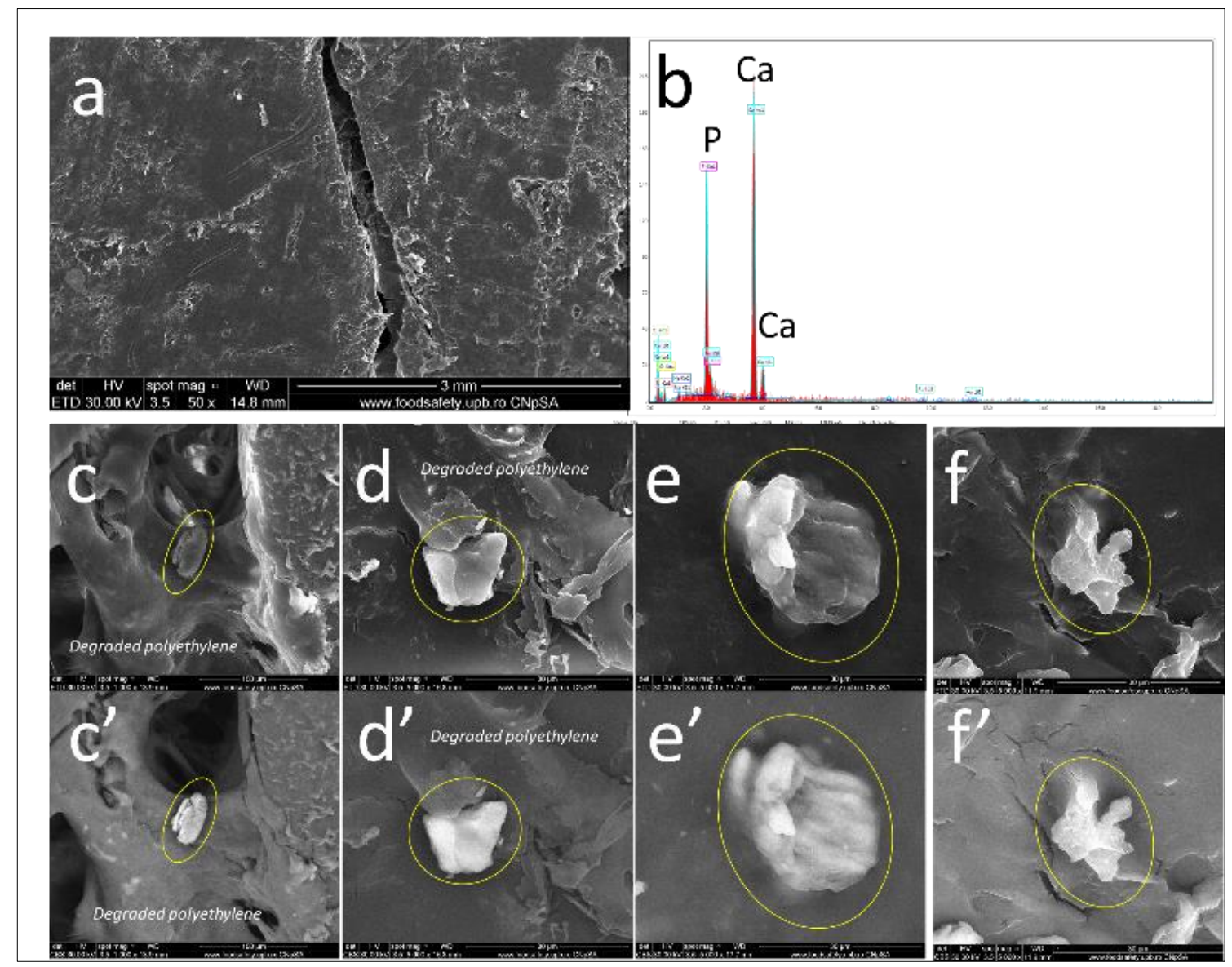

Figure 3. Representative SEM micrographs for PE wear on the femoral side of the retrieved implant: a - top view of the fracture visible in area " 4 " from the digital photograph of PE component in Figure 1 (the polymer presents superficial degradation, small particles are visible and a longitudinal fracture along the direction of the movement of the femoral head); $b$ - representative EDAX spectrum indicating a phospho-calcic nature of analyzed fragments of the bone mineral phase confirming the presence of in the analyzed fragments; different degradation areas, with fragments: $c-f$ : appearance in secondary electrons; $\mathrm{c}^{\prime}-\mathrm{f}^{\prime}$ : appearance in back scattered electrons (BSED)

Figure 4 presents various worn features specific to the tibial side of the insert: fractures and micro fractures, scratches, rough areas, delaminated areas with embedded micro particles, pitting and micro particles. Multidirectional scratches and pits are noticed in Figure 4a, multiple micro fractures in Figure $4 \mathrm{c}$ and $4 \mathrm{~d}$, fractures in Figure $4 \mathrm{e}$ and Figure $4 \mathrm{f}$ and roughness modification in Figure $4 \mathrm{~g}-\mathrm{i}$. The EDAX spectrum identified the main peak specific to carbon from PE. 

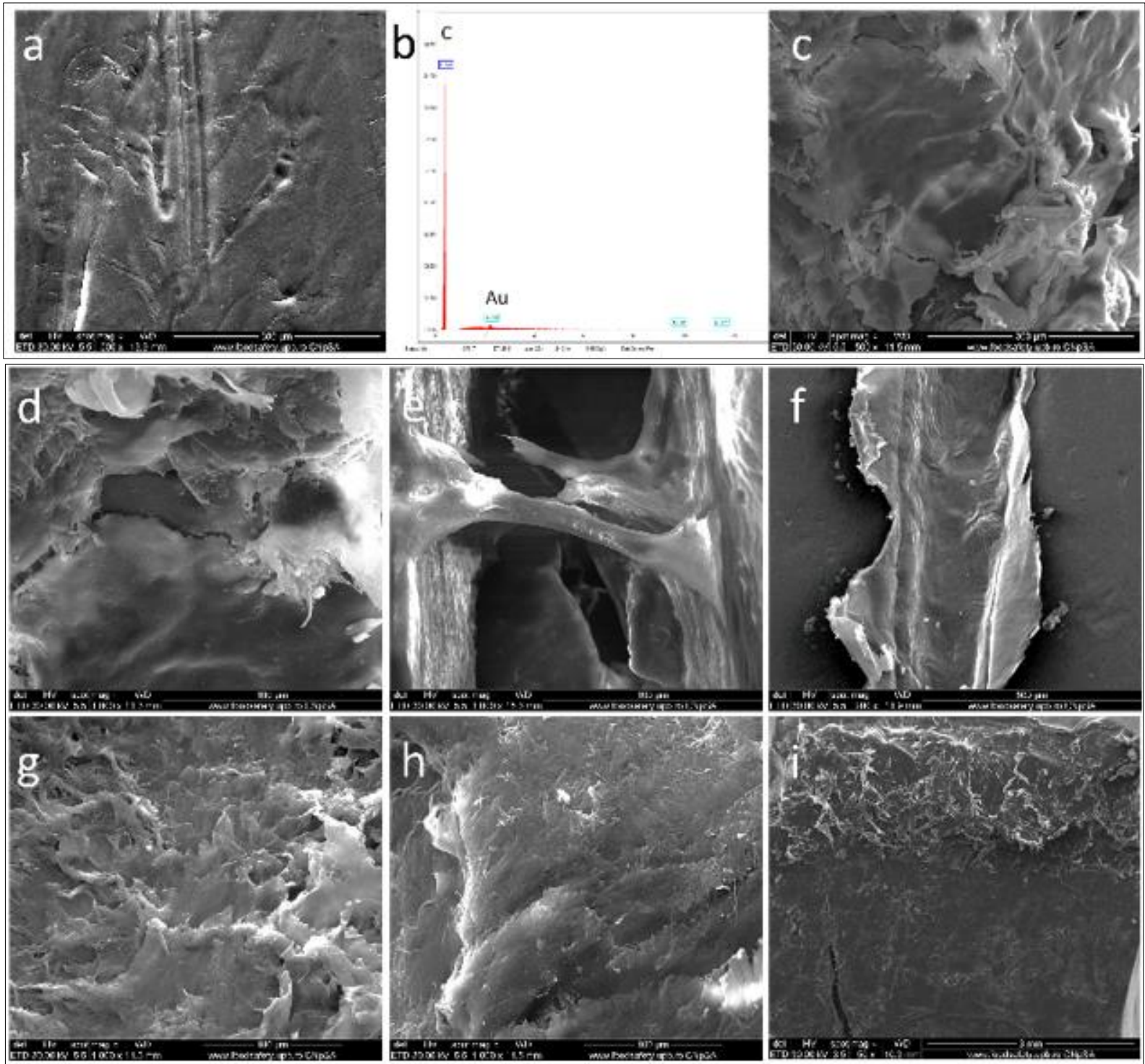

Figure 4. Representative SEM results for PE wear on the tibial side of the retrieved implant: a, c-i- micrographs; b - EDAX spectrum characteristic to PE

\subsection{ATR-FTIR spectra}

The FTIR spectrum of PE contains characteristic strong bands at around $2916 \mathrm{~cm}^{-1}, 2848 \mathrm{~cm}^{-1}(\mathrm{CH}$ stretch), $1472 \mathrm{~cm}^{-1}\left(\mathrm{CH}_{2}\right.$ deformation/ $\mathrm{CH}_{2}$ bend) and doublet at $717 \mathrm{~cm}^{-1}\left(\mathrm{CH}_{2}\right.$ rock). Since the subsurface whitening suggested possible oxidation of PE, spectra were recorded from distinct surface areas and from corresponding bulk regions. A new peak was noticed at $1719 \mathrm{~cm}^{-1}$ at the surface, while a lower signal at $1714 \mathrm{~cm}^{-1}$ was also visible in the bulk region spectrum (Figure 5a). Such vibrations are assigned to ketone resulting from oxidation. Additional interesting FTIR features were also identified in other areas of the insert at the surface, near the fractured areas. The recorded ATR-FTIR spectra indicated the presence of peaks at $1646 \mathrm{~cm}^{-1}$ and $1543 \mathrm{~cm}^{-1}$, non-specific to PE, that can be attributed to amide I and amide II vibrations. Together with the new broad and intense peak at $3282 \mathrm{~cm}^{-1}$ (assigned to $\mathrm{O}-\mathrm{H}$ and $\mathrm{N}-\mathrm{H}$ vibrations from amide A specific to proteins) these spectral modifications suggest protein contamination (Figure 5b). Since dark fragments seem inserted into the fractured regions of the PE bearing, ATR-FTIR spectra were recorded on representative samples removed from such areas. Figure $5 \mathrm{c}$ presents the specific spectral appearance, of such brown fragments obtained from area 2 of the tibial side view reprezented in Figure 2. The strong and broad peak at $3289 \mathrm{~cm}^{-1}$ is assigned to amide A vibrations, while at $3065 \mathrm{~cm}^{-1}$ an amide B peak is visible. Such vibrations are typical to proteins such as those from bone, therefore they 
suggest protein adsorption or contamination with small bone fragments migrated into the fractured area of PE (Figure 5c). The triplet at $2958 \mathrm{~cm}^{-1}, 2923 \mathrm{~cm}^{-1}$ and $2852 \mathrm{~cm}^{-1}$, respectively, corresponds to saturated $\mathrm{C}-\mathrm{H}$ stretching vibrations of combined origin. Considering the brownish color localized deep, into the fracture sites, similar to the color of bone cement from the femoral component, the ATR-FTIR spectra of retrieved cement fragments from the retrieved femoral component were also recorded, to be used as comparison. Conventional polymethyl methacrylate (PMMA) vibrations were recorded: a triplet at $2959 \mathrm{~cm}^{-1}, 2923 \mathrm{~cm}^{-1}$ and $2852 \mathrm{~cm}^{-1}$ for C-H stretching vibrations, a strong peak at $1726 \mathrm{~cm}^{-1}$ assigned to $\mathrm{C}=\mathrm{O}, 1443 \mathrm{~cm}^{-1}$ assigned to $\mathrm{O}-\mathrm{CH}_{3}$ combined with $\mathrm{CH}_{2}$ bend, $1237 \mathrm{~cm}^{-1}$ for $\mathrm{CH}_{2}$ twisting, $1141 \mathrm{~cm}^{-1}$ for $\mathrm{C}-\mathrm{O}$, and $980 \mathrm{~cm}^{-1}$ for C-O-C vibration. In addition to them, a weak and very broad peak at about 3300 $\mathrm{cm}^{-1}$ may be assigned to amide A from proteins, while amide I and amide II vibrations could be noticed at $1646 \mathrm{~cm}^{-1}$ and $1546 \mathrm{~cm}^{-1}$ on the retrieved cement. The protein-specific peaks detected on the surface of the retrieved PMMA cement suggests the cement presents adsorbed proteins or it contains proteins from the surrounding tissue due to a strong interface bonding to bone. These data suggest that the dark fragments region may present a combination with both bone and cement micro particles.

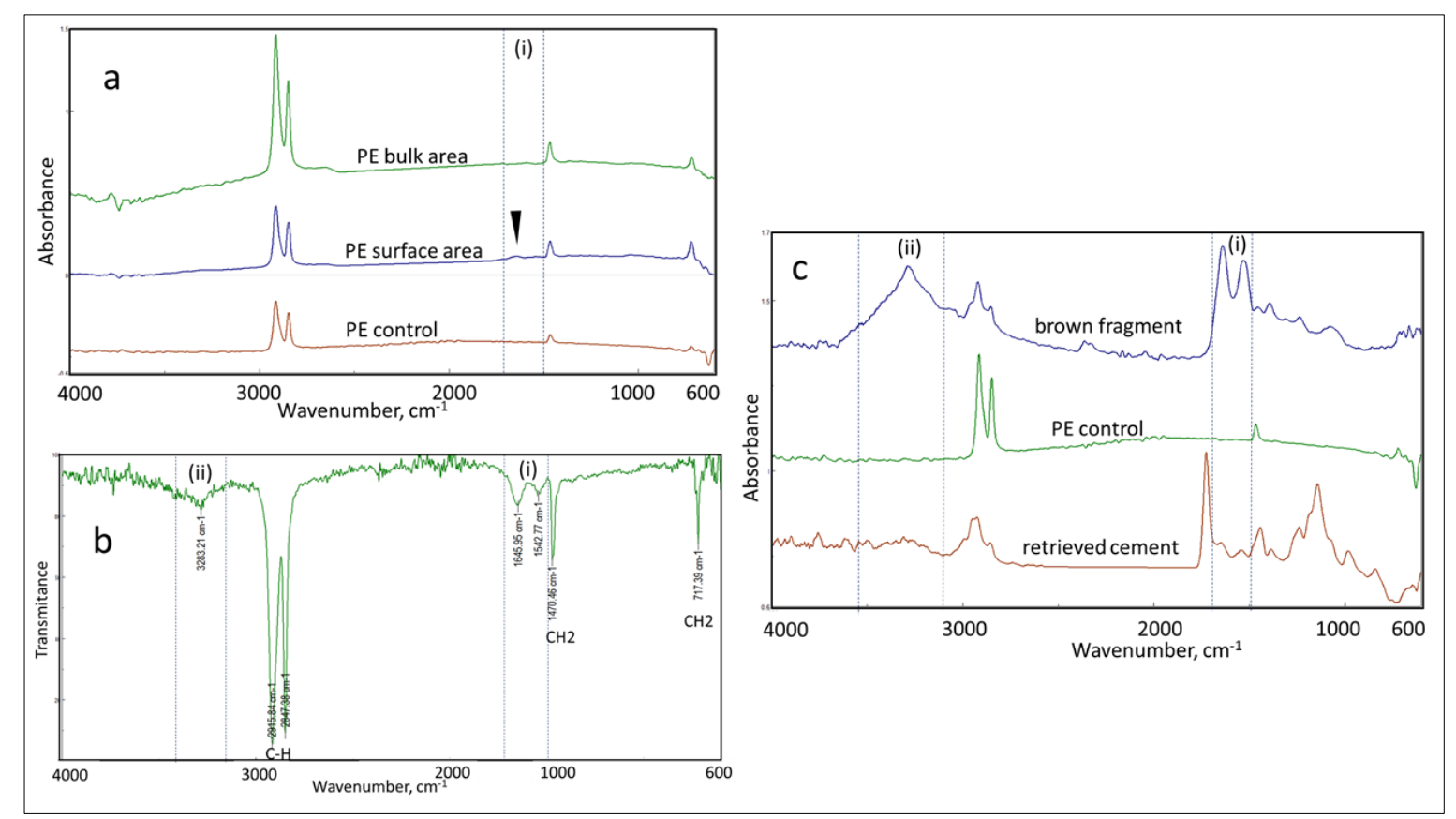

Figure 5. ATR-FTIR spectra. a - PE oxidation assessment: (i) ketone vibration; b - PE surface from the area 2 of the femoral side in Figure 1: (i) amide I, amide II; (ii) $\mathrm{OH}$, NH vibrations; c) brown fragment compared with cement and PE (i) amide I, amide II; (ii) $\mathrm{OH}$, $\mathrm{NH}$ vibrations

Mobile bearing unicompartmental knee arthroplasty is one of the treatment options for knee medial osteoarthritis, with clinical results documented in numerous reports [17, 18]. The survival rates and biomechanical performances of this type of implants have consistently improved during last twenty years [19]. However, several types of failures are documented in unicompartmental knee replacement; among them wear of PE, loosening and bearing dislocation [20-22]. In this context, it is important to understand the wear-related complications by studying and publishing the case with implant failures. For improving the performances of medical devices and technical procedures, interdisciplinary approaches are necessary, the use of material science characterizations techniques, implants design changes and the use of new manufacturing process are beneficial for the medical field [23-25].

Failure modes are depending on the implant type: mobile or fixed. Mobile implants exhibit bearing dislocation as the main failure type [26]. Reports document that mobile UKA are more often revised for bearing dislocation comparing to fixed ones which are revised mainly for polyethylene wear [27]. 
Usually, bearing dislocations is documented to take place in the first post-operative 1 to 5 years [28] and is related to inaccurate ligament balancing or inappropriate bearing height [14]. We are reporting a case with late bearing dislocation - at 11 years postoperative, in which a traumatic event may be the trigger; another particular aspect of the presented case is that the dislocated bearing was broken in two large pieces. Also, the explanted insert presented severe PE macroscopically wear consisting in pitting, scratching and delamination.

Wear patterns in unicompartmental were reported and classified; in a paper from 2010 Kendrick et al. classified the wear into 3 groups, based on macroscopically appearance of bearing, of articular surfaces and intra- or extra articular impingement [14]. Considering this classification, we can consider our case to be included in group three. In the same paper, the reported mean time until revision was 8.4 years; our case failure was reported at 12 years after surgery and it was related to a traumatic event. This is why we were looking further, searching for additional factors to explain the pattern of PE wear. The complete explant examination pointed out that inside the pitting and delamination areas, bone micro bits and cement particles were found. That leads us to the idea that intraarticular impingement, caused by bone and cement debris may be the starting point for PE wear and one of the additional factors that led to insert breakage (complication rarely found in this model of mobile bearing, ultra-congruent implant). Experimental studies in PE tibial components, show that fatigue and fracture mechanisms are directly related to the plastic flow parameters of UHMWPE, such as the yield stress and ultimate stress. The microscopic plastic flow behavior, especially in the presence of structural defects, as for example, remnants of intergrain boundaries, is considered to play an important role in the clinical performance of UHMWPE components. Also, for compression molded PE implants, the deformation instability leads to kinking of lamellae oriented initially along the loading direction [29]. This comportment may also explain the aspects found in our case.

Intraarticular bone loose bodies may occur in unicompartmental knee replacement and their potential as destructive mechanism was reported as a presumed cause of impingement and trigger for PE wear [29]. Third-body wear caused by particulate debris (bone, cartilage fragments and bone cement) is one mechanism responsible for accelerated wear and early failure of total knee arthroplasty.

To our knowledge, this is the first report documenting the bone debris inside the deep fracture of the explanted mobile bearing insert. Our result is a strong advice, suggesting that a careful lavage with saline after metal implant insertion and before PE bearing implantation may prevent these events in the future. It is sure that that postponing surgery - for at least 5 months in our case - severely increases wear and initiated femoral and tibial metallic implant deterioration; from a relatively minor insert exchange the surgery went to conversion to total knee replacement.

\section{Conclusions}

In case of severe and unexpected PE insert wear in mobile unicompartmental knee a third body mechanism must be taken in consideration. All preventive measures in order to reduce third body production inside the joint should be used at implantation time - orthopedic surgeons should be aware of wear mechanisms typical to mobile bearing implants.

The severity of wear is time depending - once diagnosed it should be properly addressed.

In real life, we can suspect that third body wear is rarely pure bone or pure cement, but a combination of the two above mentioned. The combination enhanced contribution of debris in severe PE wear initiation and progression. Complete examination of the explants exhibiting breakage or severe wear may help in understanding pathogenic ways of failure in unicompartmental knee.

\section{References}

1.WILHELM SK, HENRICHSEN JL, SILJANDER M, MOORE D, KARADSHEH M. Polyethylene in total knee arthroplasty: Where are we now? J Orthop Surg (Hong Kong). May-Aug;26(3) 2018, 23

2.EMERSON R.H., HIGGINS L.L., Unicompartmental knee arthroplasty with the Oxford prosthesis in patients with medial compartment arthritis. J. Bone Joint Surg. Am. 90A, 2008, 118-122. 
3.HOLZER L.A., HOLZER G., The most influential papers in unicompartmental knee arthroplasty. Knee Surg. Relat. Res. 32(1), 2020, 54-56.

4.MURRAY DW, GOODFELLOW JW, O'CONNOR JJ. The Oxford medial unicompartmental arthroplasty - a ten-year survival study. J Bone Joint Surg Br. 80B, 1998;983-989.

5.LIDDLE AD, JUDGE A, PANDIT H, MURRAY DW., Adverse outcomes after total and unicompartmental knee replacement in 101,330 matched patients: a study of data from the National Joint Registry. Lancet. 384, 2014, 1437-1445.

6.FABRE-AUBRESPY M, OLLIVIER M, PESENTI S, PARRATTE S, ARGENSON JN. Unicompartmental Knee Arthroplasty in Patients Older Than 75 Results in Better Clinical Outcomes and Similar Survivorship Compared to Total Knee Arthroplasty. A Matched Controlled Study. J Arthroplasty. 31(12): 2016; 2668-2671.

7.BOTTOMLEY N, JONES LD, ROUT R, ALVAND A, ROMBACH I, EVANS T, JACKSON WF, BEARD DJ, PRICE AJ. A survival analysis of 1084 knees of the Oxford unicompartmental knee arthroplasty: a comparison between consultant and trainee surgeons. Bone Joint J. 98-B(10 Supple B): 2016 Oct; 22-27.

8.PSYCHOYIOS V, CRAWFORD RW, O'CONNOR JJ, MURRAY DW. Wear of congruent meniscal bearings in unicompartmental knee arthroplasty - A retrieval study of 16 specimens. J Bone Joint Surg Br. 80B, 1998, 976-982

9.GRECU D, ANTONIAC I, TRANTE O, NICULESCU M, LUPESCU O: Failure Analysis of Retrieved Polyethylene Insert in Total Knee Replacement, Mater. Plast., 53(4), 2016: 776-780

10.***ASTM F561 - 19 Standard Practice for Retrieval and Analysis of Medical Devices, and Associated Tissues and Fluids - www.astm.org

11.FURNES O, ESPEHAUG B, LIE SA, VOLLSET SE, ENGESAETER LB, HAVELIN LI. Failure mechanisms after unicompartmental and tricompartmental primary knee replacement with cement. $\mathrm{J}$ Bone Joint Surg Am. 89A: 2007;519-525

12.PRICE AJ, SHORT A, KELLETT C, et al. Ten-year in vivo wear measurement of a fully congruent mobile bearing unicompartmental knee arthroplasty. J Bone Joint Surg [Br] 87-B 2005:1493-7.

13.COLLIER MB, ENGH CA, MCAULEY JP, ENGH GA. Factors associated with the loss of thickness of polyethylene tibial bearings after knee arthroplasty. J Bone Joint Surg 2007 89A: 1306-1314

14.KENDRICK BJ, LONGINO D, PANDIT H, SVARD U, GILL HS, DODD CA, MURRAY DW, PRICE AJ. Polyethylene wear in Oxford unicompartmental knee replacement: a retrieval study of 47 bearings. J Bone Joint Surg Br. 92(3): 2010 Mar; 367-73.

15.GHOSH P, MOHAMMAD HR, MARTIN B, CAMPI S, MURRAY DW, MELLON SJ. Low polyethylene creep and wear following mobile-bearing unicompartmental knee replacement. Knee Surg Sports Traumatol Arthrosc. 2020 Sep 17. doi: 10.1007/s00167-020-06243-7.

16.KOP AM, PABBRUWE MB, KEOGH C, SWARTS E. Oxidation of Second Generation Sequentially Irradiated and Annealed Highly Cross-Linked X3 ${ }^{\mathrm{TM}}$ Polyethylene Tibial Bearings. J Arthroplasty. 30(10): 2015 Oct; $1842-6$

17.JOHAL S, NAKANO N, BAXTER M, HUJAZI I, PANDIT H, KHANDUJA V Unicompartmental Knee Arthroplasty: The Past, Current Controversies, and Future Perspectives. J Knee Surg. 31(10) 2018 Nov:992-998

18.KAHLENBERG CA, RICHARDSON SS, GRUSKAY JA, CROSS MB Trends in utilization of total and unicompartmental knee arthroplasty in the United States. J Knee Surg . (2020) doi: 10.1055/s-00401702189

19.KHOW YZ, LIOW MHL, LEE M, CHEN JY, LO NN, YEO SJ. The effect of tibial and femoral component coronal alignment on clinical outcomes and survivorship in unicompartmental knee arthroplasty. Bone Joint J. 103-B (2): 2021 Feb; 338-346.

20.SABAH SA, LIM CT, MIDDLETON R, VON FRITSCH L, BOTTOMLEY N, JACKSON WFM, PRICE AJ, ALVAND A. Management of aseptic failure of the mobile-bearing Oxford unicompartmental knee arthroplasty. Knee. 27(6) 2020 Dec:1721-1728 
21.PONTOH LA, DILOGO IH, HARTONO F, RHATOMY S, FIOLIN J. Meniscal bearing dislocation following minimally invasive Oxford medial unicompartmental knee arthroplasty treated with simple open reduction: Case report. Int J Surg Case Rep. 792021 Feb:371-374.

22.YANG I, HAMILTON TW, MELLON SJ, MURRAY DW. Systematic review and meta-analysis of bearing dislocation in lateral meniscal bearing unicompartmental knee replacement: Domed versus flat tibial surface. Knee. 28 2021 Jan:214-228.

23.AITCHISON, G. A., HUKINS, D. W., PARRY, J. J., SHEPHERD, D. E., \& TROTMAN, S. G. A review of the design process for implantable orthopedic medical devices. The Open Biomedical Engineering Journal, 3, 2009: 21-27.

24.MARINESCU, R., POPESCU, D., LAPTOIU, D. A Review on 3D-Printed Templates for Precontouring Fixation Plates in Orthopedic Surgery, Journal of Clinical Medicine, J. Clin. Med. 9(9) 2020, 29-08;

25.JONES L.C., TSAO A.K., TOPOLESKI L.D.T. Orthopedic Implant Retrieval and Failure Analysis. In: Eliaz N. (eds) Degradation of Implant Materials. Springer, New York, NY. (2012)

26.MURRAY DW, LIDDLE AD, DODD CA, PANDIT H. Unicompartmental knee arthroplasty: is the glass half full or half empty? Bone Joint J. 97(10) 2015:3-8

27.Z. ABU AL-RUB, J.N. LAMB, R.M. WEST, X. YANG, Y. HU, H.G. PANDIT, Survivorship of fixed vs mobile bearing unicompartmental knee replacement: A systematic review and meta-analysis of sixty-four studies and National Joint Registries, The Knee, 27, Issue 5, 2020, 1635-1644

28.BERGESON AG, BEREND KR, LOMBARDI AV JR, HURST JM, MORRIS MJ, SNELLER MA. Medial mobile bearing unicompartmental knee arthroplasty: early survivorship and analysis of failures in 1000 consecutive cases. J Arthroplasty. 28(9 Suppl) 2013 Oct:172-5.

29.SCHROEDER S, BRAUN S, MUELLER U, SCHROEDER M, SONNTAG R, JAEGER S, JAN PHILIPPE KRETZER, Polyethylene wear and metal release of TiNbN-coated knee implants, Wear, Volumes 458-459, 2020, 203426.

Manuscript received: 17.09 .2021 